\title{
Bayesian Calibration of AquaCrop Model for Winter Wheat by Assimilating UAV Multi-Spectral Images
}

\author{
Tianxiang Zhang ${ }^{\mathrm{a}}$, Jinya Su ${ }^{\mathrm{b}, *}$, Cunjia Liu ${ }^{\mathrm{a}}$, Wen-Hua Chen ${ }^{\mathrm{a}}$ \\ ${ }^{a}$ Department of Aeronautical and Automotive Engineering, Loughborough University, Loughborough, LE11 3TU, U.K. \\ ${ }^{b}$ School of Computer Science and Electronic Engineering, University of Essex, Colchester, CO4 3SQ, U.K.
}

\section{Abstract}

Crop growth model plays a paramount role in smart farming management, which not only provides quantitative information on crop development but also evaluates various management strategies. A reliable model is desirable but challenging due to the presence of unknown and uncertain parameters; therefore, crop model calibration is significant to achieve its potentials. This work is focused on the calibration of AquaCrop model by leveraging advanced Bayesian inference algorithms and UAV multi-spectral images at field scales. In particular, aerial images with high spatialtemporal resolutions are first applied to obtain Canopy Cover (CC) value by using machine learning based classification. The CC is then assimilated into AquaCrop model and uncertain parameters could be inferred by Markov Chain Monte Carlo (MCMC). Both simulation and experimental validation are performed. The experimental aerial images of winter wheat at Yangling district from Oct/2017 to June/2018 are applied to validate the proposed method against the conventional optimisation based approach by Simulated Annealing (SA). 100 Monte Carlo simulations show that the root mean squared error (RMSE) of Bayesian approach yields a smaller parameter estimation error than optimisation approach. While the experimental results show that: (i) a good wheat/background classification result is obtained for the accurate calculation of CC; (ii) the predicted CC values by Bayesian approach are consistent with measurements by 4 -fold cross validation, where the RMSE is 0.0271 smaller than optimisation approach (0.0514); (iii) in addition to parameter estimation, their distribution information is also obtained in the developed Bayesian approach, reflecting the prediction confidence. It is believed that the Bayesian model calibration, although is developed for AquaCrop model, can find a wide range of applications to various simulation models in agriculture and forestry.

Keywords: Unmanned Aerial Vehicle (UAV); Multispectral image; Machine learning; Model calibration; Bayesian inference

\section{Introduction}

Agricultural crop states are paramount for smart farming management and food security. A timely and accurate estimation of canopy states has become an effective approach for crop monitoring, irrigation decision-making and yield management [1, 2]. In this regard, a reliable crop model is desirable for crop state estimation. However, due to the presence of unknown and uncertain parameters in spatial distribution of soil properties and crop parameters, the prediction performance of crop model degrades significantly if model parameters are chosen inappropriately [3].

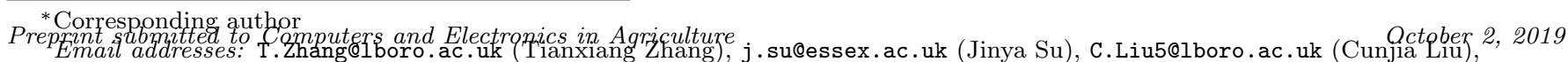
W.Chen@lboro.ac.uk (Wen-Hua Chen)
} 
Consequently, model parameters need to be calibrated before its potentials being realised. To this end, the integration of crop models and remote sensing data are drawing ever-increasing research interest 4]. It is noted that the accuracy of remote sensing data plays an important role in enhancing the predictive power of the calibrated model. Therefore, remote sensing data of high spatial/temporal resolutions is desirable, and so UAV remote sensing is preferable in this regard.

Crop model, quantitatively simulating crop physiological development, is defined by mathematical formulations driven by carbon, water, and light 4, 5. Various crop growth models are developed for various semantic applications in the literature [6] such as World Food Studies (WOFOST), Crop Estimation through Resource and Environment Synthesis-Wheat (CERES-Wheat), Decision Support System for Agro-technology Transfer-Cropping System Model (DSSAT), APSIM, STICS, CropSyst and AquaCrop model. In particular, AquaCrop model [7, a water-driven crop model, possesses a number of fine properties over others in terms of simplicity, robustness and accurateness. Therefore, this model is drawing increasing attention in precision agriculture applications such as crop monitoring, irrigation management and yield prediction [8, 9].

In terms of remote sensing data, different sensing platforms equipped with different sensors of various spatial/spectral resolutions are available in the literature such as satellite/manned-aircraft based [10] and Unmanned Aerial Vehicles (UAV) based ones [1]. Satellite/manned-aircraft remote sensing, although is suitable for large-scale applications, is usually constrained by its poor spatial resolution in farm-scale applications. UAV remote sensing, however, is of low-cost, with a high resolution and good flexibility, and therefore has become an important complement to conventional remote sensing. It has been extensively applied to smart agriculture at field scales such as stress (e.g. disease, weed, drought) monitoring and crop parameter estimation [12, 13, 14].

In crop model calibration, the measurements are usually chosen as the easily-accessible dynamic states such as Leaf Area Index (LAI) in WOFOST [15], leaf nitrogen accumulation in WheatGrowth model [16], biomass and CC in AquaCrop [17]. As a key crop growth parameter, $\mathrm{CC}$ denotes the canopy percentage, which is defined as the fraction between plant foliage projection to horizontal surface and total ground area [18]. CC calculation, therefore, could be formulated as an image segmentation problem, where the pixels are classified into two classes including wheat and non-wheat. The proportion of wheat pixels in a given area can be treated as CC value. The commonly used approaches for CC calculation are threshold based and machine learning based approaches [19. Threshold approach relies on a threshold of particular band or index [11. This approach is relatively simple, however, is sensitive to environmental variations [20]. Machine learning approach instead relies on labelled data to segment the images without the requirement of a threshold. This approach usually results in better performance although at the expense of a relatively high computation/labelling workload [19]. Considering that computation cost is not a concern for offline crop model calibration, machine learning based approach is adopted in this work due to its better performance.

The emerging model calibration methods integrating crop models and remote sensing data have become an effective 
approach for estimating crop parameters and simulating crop dynamics. The dominant approach in the literature is optimization based model calibration 4. In this approach, various optimisation algorithms are drawn to calibrate the model parameters by minimising the fitness (error) function, which is defined by the discrepancy between measurement data and predicted output by the model [4. For example, particle swarm optimization (PSO) is adopted in [1] to calibrate AquaCrop model by using historical remote sensing data, based on which biomass and final yield are predicted before harvest. Moreover, other optimisation algorithms have also been employed such as simplex search algorithm, Least Squares Method (LSM), Genetic Algorithm (GA), Shuffled Complex Evolution (SCE-UA) [21, 22, 23. The accuracy of SCE-UA is shown to be better than others, however, these algorithms may still easily get stuck in a local minima due to the complexity of the optimisation problem at hand. In addition, only a point estimate is returned in optimization approaches with no confidence information.

Different from optimization approaches, Bayesian approach can infer the posterior distribution of uncertain parameters based on available information. The main idea of Bayesian calibration is to derive the posterior distribution of model parameters of interest by integrating the prior information and measurement information by Bayesian rule. The literature on Bayesian calibration for agricultural applications is sparse compared against optimization approaches. Still natural history model and forest model are calibrated in [24, 25] respectively, where the uncertain parameters are estimated by applying Markov Chain Monte Carlo (MCMC) algorithm. However, this approach has received little attention in the community of smart farming, which is the main aim of this study.

Winter wheat is one main crop in China (north China in particular), and therefore improving crop model simulation accuracy is significant for addressing the challenges in smart farming such as dynamic states prediction, irrigation management and yield prediction prior to harvest. Previous studies are mainly focused on optimisation approaches by using satellite or ground sensing data. In this approach, only point estimate of model parameters is available, where the confidence of the estimate is missing. However, very little literature information is available on model parameter estimation by Bayesian approach, particularly by assimilating UAV multispectral imagery at field scales. Consequently, the aim of this study is to calibrate AquaCrop model by assimilating UAV multi-spectral aerial imagery using Bayesian calibration. The developed approach is compared against the conventional optimisation based approach (e.g. simulated annealing in particular), where both Monte Carlo (MC) simulation and experimental verification are conducted. The main contributions of this work are summarized:

(1) State-of-the-art UAV multi-spectral image by RedEdge camera and DJI S1000 UAV are drawn to work out the key measurement variable (CC) of AquaCrop model by machine learning classification;

(2) Bayesian inference is drawn to integrate the AquaCrop model and remote sensing measurements so that the posterior distribution (instead of point estimate) of AquaCrop model parameters is obtained;

(3) Both Monte Carlo simulation and experimental validation are performed to verify the developed Bayesian calibration against conventional optimization based approach, where a promising result is obtained in term of model 
parameter estimation and CC prediction.

\section{Materials}

In this section, materials related to the experimental work in this study are introduced, which mainly consist of the experiment site for winter wheat and UAV-camera system for multi-spectral image acquisition.

\subsection{Experiment fields}

The experiment was conducted in Caoxinzhuang experiment field (latitude: $34^{\circ} 306^{\prime} N$, longitude: $108^{\circ} 090^{\prime} E$, $499 \mathrm{~m}$ a.s.l.), which belongs to Northwest A\&F University located in Yangling city, Shannxi Province, China (see Fig 1 for the location). The soil property in this study is loessal soil with organic content of $8.0 \%-15.0 \%$. The climate in the experimental region is characterized by semi-humid and semi-arid with a mean annual temperature, precipitation of $12.9^{\circ} \mathrm{C}, 635 \mathrm{~mm}$ (especially from June to September), respectively.

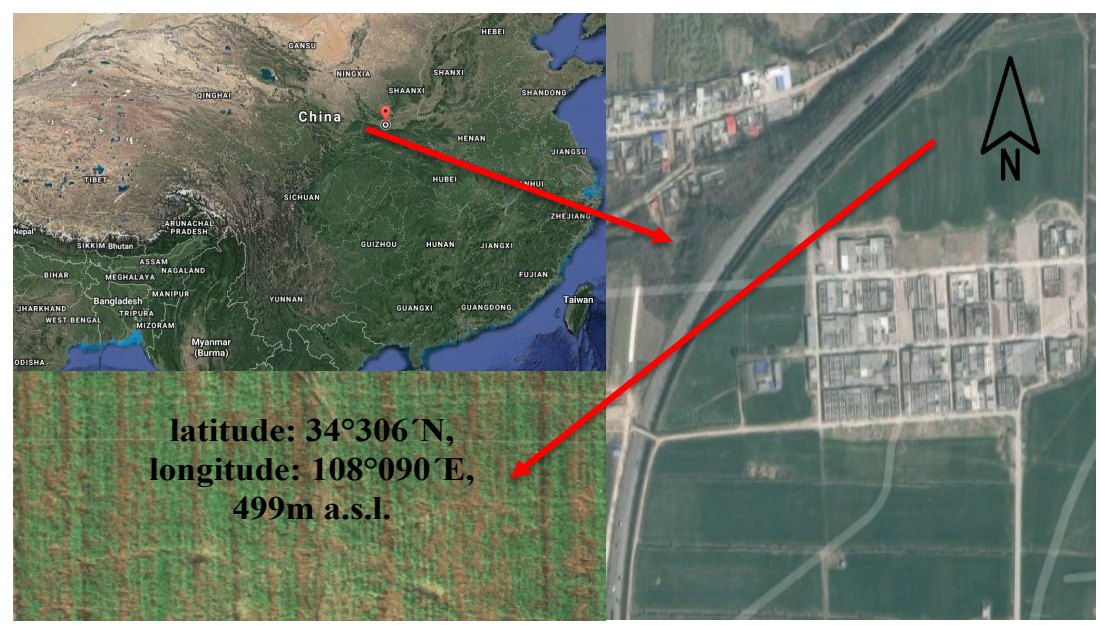

Figure 1: Geographic location of the experimental field for winter wheat.

In this study, one cultivars named Xiaoyan 22 developed by Northwest A\&F university was selected and planted at a line spacing of $16 \mathrm{~cm}$ with a rate of $30 \mathrm{~g}$ seeds $/ \mathrm{m}^{2}$ from 5/October $/ 2017$ to early June, 2018. Local standard practise was implemented for field management, in addition, twice irrigation was carried out on 10/December/2017 and 13/March/2018 with no fertilizer. The meteorological data (one key input information of the AquaCrop model) can be downloaded from National Meteorological Information Center (http://data.cma.cn) and the basic soil data are also available on national Earth system Science Data Sharing Infrastructure (http://www.geodata.cn).

\subsection{Multispectral aerial image}

The area of the field is $5 \mathrm{~m}$ by $10 \mathrm{~m}$ and was investigated from 11/December/2017 to 23/May/2018, where eight UAV surveys were conducted to collect the aerial images. In this study, a five-band multi-spectral camera named RedEdge (MicaSense Company, Seattle, USA) is equipped on the commercial DJI Spreading Wings S1000 Octocopter 
(DJI Company, Shenzhen, China) (see Fig 2). RedEdge camera outperforms conventional RGB camera in that: (1) RedEdge camera possesses extra Rededge and NIR bands, providing extra spectral information for vegetation classification; (2) calibration panel is adopted to calibrate the multispectral images, as a result, it is more robust against environmental (illumination) variations. The specifications of the UAV is referred to [26] and the weight, dimensions, image resolution of RedEdge camera are $135 \mathrm{~g}, 5.9 \mathrm{~cm} \times 4.1 \mathrm{~cm} \times 3.0 \mathrm{~cm}$ and $1280 \times 960$ pixels, respectively.
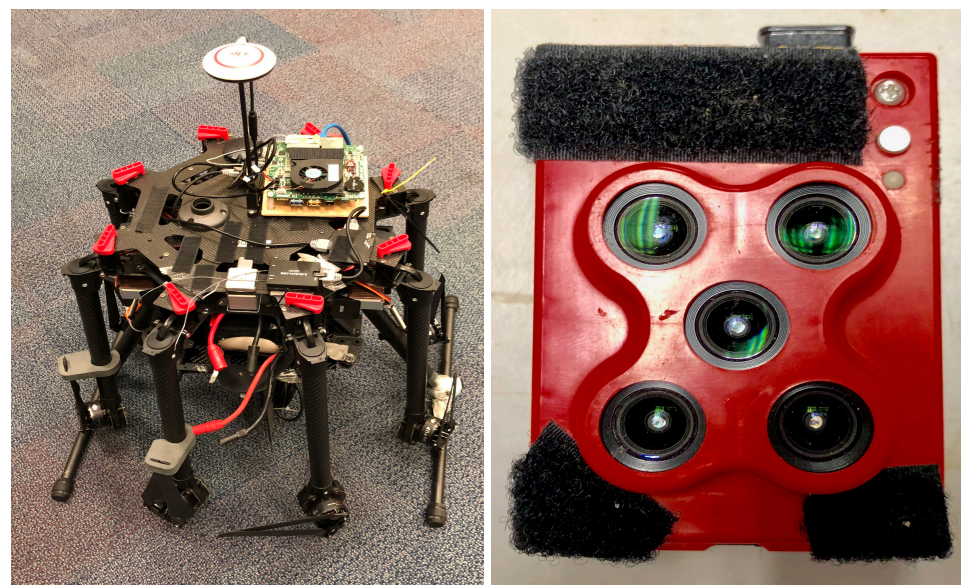

Figure 2: DJI S1000 with RedEdge Camera

In addition, RedEdge camera is fixed on a gimbal to attenuate the adverse effects of wind, so that high-quality images can be captured during the survey. The spectral information of RedEdge camera is displayed in Table 1 . Multispectral images were obtained on winter wheat key developmental stages including tillering stage (11/December/2017 and 28/December/2017), green-up stage (23/March/2018), jointing stage (01/April/2018 and 17/April/2018), anthesis stage (07/May/2018) and grain filling stage (15/May/2018 and 23/May/2018), respectively [27]. Each UAV aerial image is with the necessary information for camera calibration and image stitching. An image of a reflectance calibration panel was taken (at about $1 \mathrm{~m}$ height) before and after each flight and used in the process of image calibration to account for the side effects of environmental variations. In addition, commercial Pix4Dmapper software of version 4.2.27 is adopted to generate calibrated and georeferenced spectral reflectance data for CC calculation. The detailed process is omitted and can be referred to Section 2.3 of [1]

Table 1: Spectral information of the RedEdge camera.

\begin{tabular}{ccccc}
\hline Band No. & Name & Center Wavelength & Bandwidth & Panel reflectance \\
\hline 1 & Blue & $475 \mathrm{~nm}$ & $20 \mathrm{~nm}$ & 0.57 \\
2 & Green & $560 \mathrm{~nm}$ & $20 \mathrm{~nm}$ & 0.57 \\
3 & Red & $668 \mathrm{~nm}$ & $10 \mathrm{~nm}$ & 0.56 \\
4 & NIR & $840 \mathrm{~nm}$ & $40 \mathrm{~nm}$ & 0.51 \\
5 & RedEdge & $717 \mathrm{~nm}$ & $10 \mathrm{~nm}$ & 0.55 \\
\hline
\end{tabular}




\section{Methodologies}

In this section, the methodologies in this study are presented including CC calculation, wheat crop model and Bayesian calibration approach.

\subsection{CC calculation}

The calculation of CC is first discussed. In this study, UAV remote sensing data (e.g. five-band multispectral image) is preferred due to its high spatial/spectral resolutions. The overall process is displayed in Fig 3 , where each element is detailed in the following subsections.

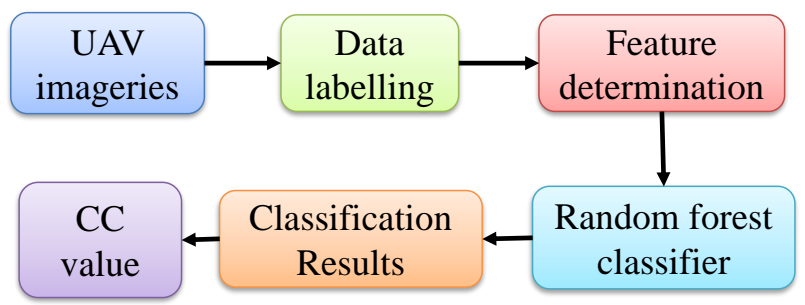

Figure 3: Overall framework for the canopy cover calculation.

\subsubsection{Data labelling and spectral analysis}

In this study, CC calculation is formulated as a wheat/non-wheat two-class classification problem so that wheat pixel proportion can be calculated for the region of interest. One specific image acquired on 11/December/2017 is used as an illustration example. It is well known that supervised classification relies on labelled data for its training, which include wheat and non-wheat pixels in this study. In this work, wheat/non-wheat pixels are directly labelled for the five-band multispectral images in Matlab environment, where a sample image is displayed in Fig 4
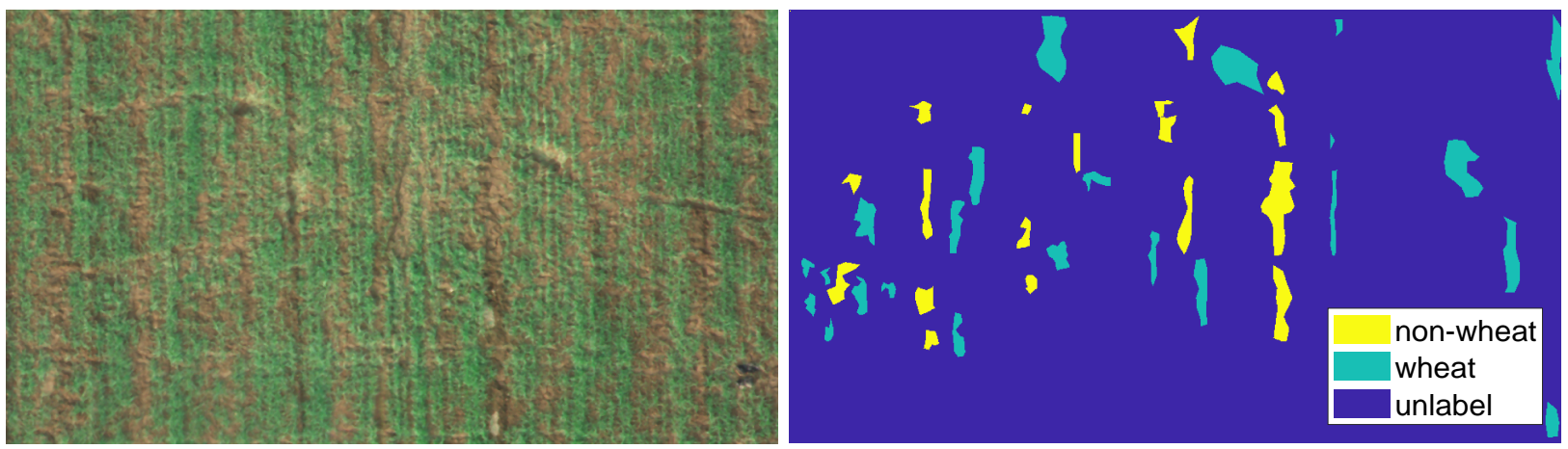

Figure 4: Original (left) and labelled (right) winter wheat on 11/Dec/2017

The spectral characteristics of the wheat/non-wheat pixels are also analysed, where the mean spectral reflectance values are shown in Fig 5. It can been seen that the green peak phenomenon is observed for wheat (green) crop where the value of Green band is higher than that of Blue and Red bands. In addition, wheat pixels also have a higher NIR 

the performance.

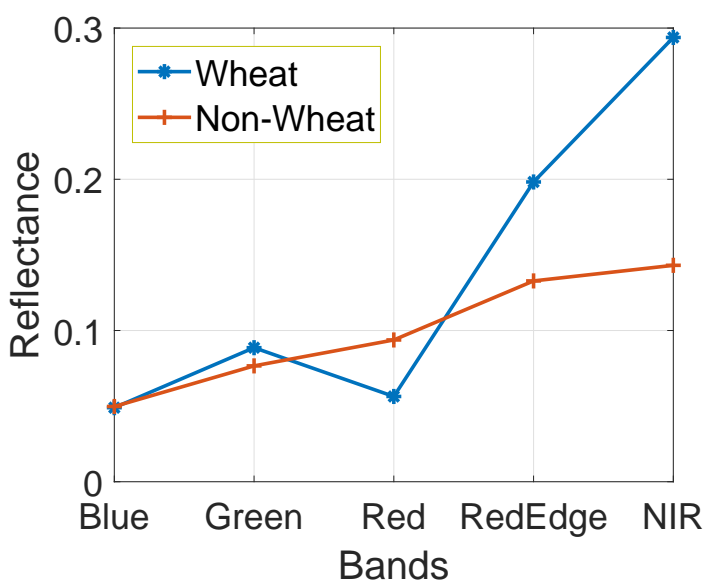

Figure 5: Average spectral reflectance value for two classes over five bands.

reflectance value than non-wheat pixels. The spectral differences provides important information for discriminating wheat pixels from non-wheat pixels. Considering that there are only five spectral bands in the multispectral images, all available bands are used as the features for the classification task in Section 3.1.2. If a large number of (redundant) features are available, feature selection approaches in [28] can be drawn to reduce the computation load while preserving

\subsubsection{Image classification}

Given labelled data in Section 3.1.1, a classifier is then required to perform the classification task so that new aerial images can be automatically classified for CC calculation. A number of classifiers can achieve this task such as Support Vector Machines (SVMs), neural network, nearest neighbour [10. In this study, random forest classifier is employed due to its high efficiency and accuracy, where the hyper-parameters are further automatically tuned by Bayesian optimization [11]. The detailed algorithm is omitted, which is referred to [11, 29].

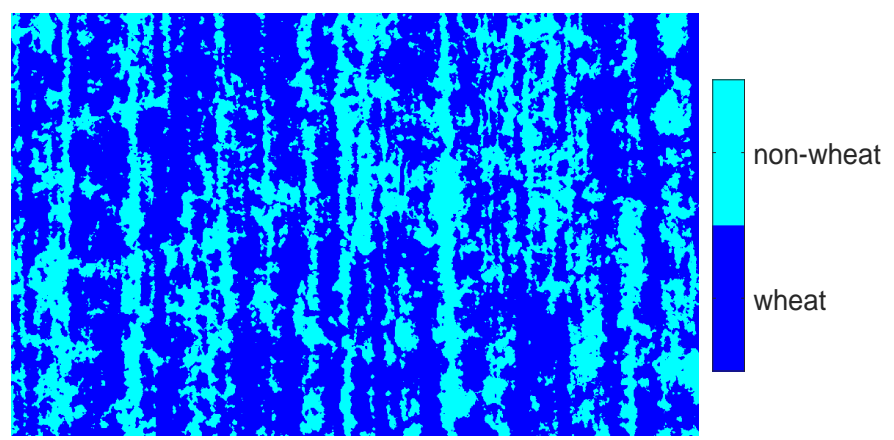

Figure 6: Classification map by the random forest classifier.

In this work, $70 \%$ and $30 \%$ of the labelled pixels are for training and testing respectively, where the classification accuracy is calculated on testing dataset. The overall classification accuracy for the example image is $99 \%$. The trained model is then applied to the original image, where the classification map is shown in Fig 6. Then CC value 
can be calculated by the formula in $\mathrm{Eq} 1$.

$$
C C=w p /(w p+n w p)
$$

Table 2: Classification accuracy and canopy cover values over time

\begin{tabular}{ccc}
\hline Acquisition Date & Overall Accuracy (\%) & CC Measurement Value \\
\hline $11 / 12 / 2017$ & 99 & 0.5896 \\
$28 / 12 / 2017$ & 99.2 & 0.7182 \\
$23 / 03 / 2018$ & 99.5 & 0.8983 \\
$01 / 04 / 2018$ & 99.3 & 0.9319 \\
$17 / 04 / 2018$ & 99.6 & 0.9225 \\
$07 / 05 / 2018$ & 99.2 & 0.9124 \\
$15 / 05 / 2018$ & 98.8 & 0.8726 \\
$23 / 05 / 2018$ & 99.1 & 0.8155 \\
\hline
\end{tabular}

\subsection{Bayesian calibration for AquaCrop model}

This section further discusses crop growth model and calibration method. The overall framework of the developed Bayesian calibration for AquaCrop model is shown in Fig 7, which include AquaCrop function, Markov Chain Monte Carlo (MCMC) method and result analysis. In this work, the CC measurement in Section 3.1 is chosen as observation variable. Different elements of the proposed framework are detailed in the following sections.

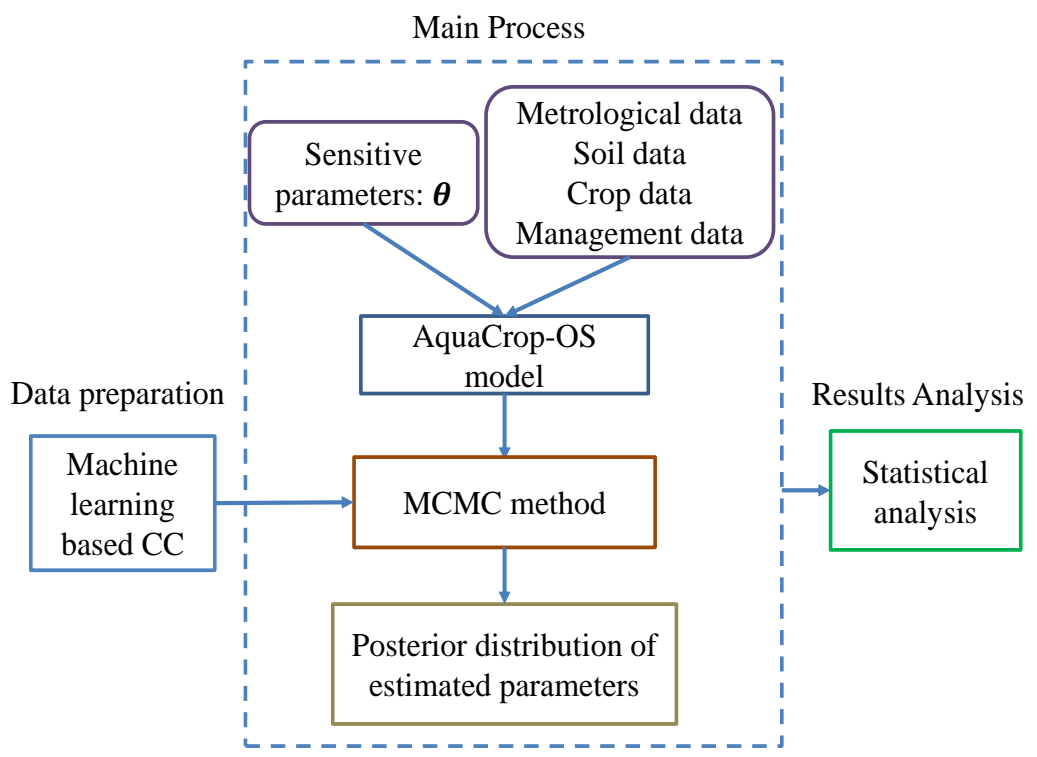

Figure 7: Framework of the proposed Bayesian calibration approach for AquaCrop model. 


\subsubsection{AquaCrop-OS model}

AquaCrop crop growth model is developed in [7, which makes a good balance between model complexity and model accuracy. It is a water balance based crop model simulating the interactions between weather, soil and crop growth. In particular, the $\mathrm{CC}$ determines the water transpiration amount at expansion, ageing, conductance and senescence stage, thus affecting the biomass production 30 . The crop's daily aboveground biomass is generated by normalised crop water productivity $\left(W P^{*}\right)$ from AquaCrop model. Biomass yield was determined by $W P^{*}$ and the ratio of crop transpiration $\left(E T_{i}\right)$ and reference evapotranspiration $\left(E T_{0 i}\right)$ via $\mathrm{Eq} 2$ and grain yield $(Y)$ is obtained by multiplying the harvest index $(H I)$ by the biomass $\left(B_{i}\right)$ as in $\mathrm{Eq} 3$

$$
B_{i}=W P^{*} \times \sum_{i=1}^{N} \frac{E T_{i}}{E T_{0 i}},
$$

$$
Y=B_{i} \times H I,
$$

where $W P^{*}$ is the normalised crop water productivity in $\mathrm{g} / \mathrm{m}^{2} ; E T_{i}$ is daily crop transpiration in $m m ; E T 0_{i}$ is the daily reference evapotranspiration in $m m ; B_{i}$ is the cumulative biomass at $i$ th day $(t o n / h a) ; H I$ is the harvest index; and $Y$ is grain yield $($ ton $/ h a)$ at time $i$.

To facilitate the model application, an open-sourced version (named AquaCrop-OS model) was later developed in Matlab environment 8 . This open-sourced model can be easily integrated with other approaches for various applications [9]. From a mathematics perspective, the dynamic system of AquaCrop model is a Markov process, where the future status at $t+1$ is only conditional on the current status at $t$ rather than the past states [31]. Therefore, the model could be simplified into Eq4

$$
\begin{aligned}
& X_{t+1}=F\left(X_{t}, \theta\right), \\
& Y_{t}=G\left(X_{t}\right)+\xi_{t}, \text { with } \xi_{t} \sim N(0, \sigma)
\end{aligned}
$$

where $F($.$) represents the crop model operator and X$ presents the canopy states (e.g. biomass, canopy cover, root depth) on each simulated date. $G($.$) denotes the measurement model with measurement noise \xi$ being with zero mean and a proper covariance $\sigma$.

\subsubsection{Bayesian calibration method}

Bayesian estimation theory: the goal of Bayesian estimation is to update the probability distribution of the sensitive parameters by integrating observation and prior [25]. Different from optimisation approach which derives parameter estimation by minimizing the predefined objective function, Bayesian calibration derives the parameter posterior distribution. 32. In particular, the posterior distribution $P(\theta \mid Y)$ is proportional to the prior parameter distribution $P(\theta)$ times the measurement likelihood function $P(Y \mid \theta)$, which is given by

$$
P(\theta \mid Y) \propto P(\theta) \times P(Y \mid \theta),
$$


where $Y$ is the observational data and $\theta$ represents the parameters to be estimated. To simplify the problem, the likelihood function is defined as the error between observations and simulated model outputs (see Eq 5). More details is given in Section 3.3

$$
P(Y \mid \theta)=P(E=Y-F(\theta))
$$

where $F($.$) denotes the function of crop model conditional on parameter \theta, E$ means the error.

Markov Chain Monte Carlo (MCMC): The MCMC process can effectively approximate the posterior distribution function (PDF). The fundamental principle of Markov Chain is that the current sample value (at time $t$ ) is based on the past sample (at time $t-1$ ), where determines whether the candidate is accepted or not with a probability. The Monte Carlo (MC) sampling method is implemented to accurately evaluate the posterior PDF for the parameters $\theta$. The main purpose of MCMC is to generate a Markov Chain with a stable distribution of the target distribution. This method can gather a series of samples at random walk generating a Markov Chain for the goal of parameter distribution. Finally, one coverage chain with accepted parameters value will be achieved at an equilibrium status. Several sampling methods have been proposed to accept or reject new states, the most popular one is Metropolis Hastings (MH) sampling method [24, 25, 33.

Delaying Rejection Adaptive Metropolis (DRAM): To increase MH sampling performance, two variants of $\mathrm{MH}$ algorithm named delaying rejection (DR) and adaptive metropolis (AM) were proposed. DR is capable of modifying the standard MH algorithm to improve the estimation efficiency as this method employs considerable given proposals and keep the reversibility in different stages [34. In AM method, the covariance matrix of the Gaussian proposal distribution is adjusted during the operation using the past chain. It can be demonstrated that the ergodicity properties of the resulted samples still exist. AM is good at creating a Gaussian proposal distribution from the current point in MCMC by computing the covariance matrix of the chain. The illustration of DRAM is given in Algorithm 1 , where $s_{d}$ is a parameter that only relies on the state space dimension $d$ where equilibrium is defined and $\varepsilon$ is a constant bigger than zero. $I_{d}$ denotes the $d$ dimensional identify matrix. $t_{0}$ denotes the initial non-adaptation time and $C_{0}$ is defined by our prior of the proposal covariance 34. The combination of DR and AM can increase the candidate acceptance probability and effectively improve the efficiency reaching to Markov Chain equilibrium. The proof of DRAM realization is referred to [34, 35. 


\section{Algorithm 1: DRAM Algorithm}

1 Initialization: Randomly select the initial parameters $\theta^{0}$ for a chain length of $M$ based on a symmetric transition kernel.

\section{Iteration: $\mathrm{i}=1$}

\section{Sampling:}

for $i=0$ to $M-1$

Construct Gaussian proposal

$$
\text { proposal mean }=\text { current } \theta
$$

proposal covariance:

if $i<i_{0}, C_{t}=C_{0}$

else

$$
C_{t}=s_{d} \operatorname{Cov}\left(\theta^{0}, \theta^{1}, \ldots \ldots . . \theta^{i-1}\right)+s_{d} \varepsilon I_{d}
$$

Randomly select the first stage proposal candidate parameter $\theta^{*}$

$$
\begin{aligned}
& \text { Sample } u \sim U[0,1] \\
& \text { If } u<\alpha=\min \left\{1, \frac{P\left(\theta^{*}\right) P\left(y \mid \theta^{*}\right)}{P\left(\theta^{i-1}\right) P\left(y \mid \theta^{i-1}\right)}\right\} \\
& \theta^{i}=\theta^{*}
\end{aligned}
$$

otherwise

Construct the second stage proposal $\theta^{* *}$

Sample $u \sim U[0,1]$

$$
\begin{aligned}
& \text { If } u<\alpha=\min \left\{1, \frac{P\left(\theta^{*}\right) P\left(y \mid \theta^{*}\right)}{P\left(\theta^{i-1}\right) P\left(y \mid \theta^{i-1}\right)}\right\} \\
& \theta^{i}=\theta^{* *}
\end{aligned}
$$

otherwise

$$
\theta^{i}=\theta^{i-1}
$$

$\mathrm{i}=\mathrm{i}+1$

\section{Return to step 2}




\subsection{Model calibration implementation}

DRAM method is implemented to obtain the crop parameter distribution by using remotely sensed data and AquaCrop model in both theoretical and experimental way. The error distribution is assumed to Gaussian with zero mean and a proper variance, thereby the likelihood function in this study is formulated as

$$
p(Y \mid \theta)=\prod_{i=1}^{N} \frac{1}{\sqrt{2 \pi \sigma^{2}}} \exp \left\{\frac{\left(y_{i}-\hat{y}_{i}\left(x_{k}, \theta\right)\right)^{2}}{2 \sigma^{2}}\right\},
$$

where $N$ is the total observation number and the $y_{i}-\hat{y}_{i}\left(x_{k}, \theta\right)$ is the error between the measurement of dynamic states $y_{i}$ and modelled states value by employing the crop model operator $F($.$) . The variance \left(\sigma^{2}\right)$ can be predefined or estimated along with model parameters [35].

\section{Systematic validation}

In this section, different model validation approaches including Monte Carlo simulation and real-world experiments are conducted to assess the performance of the developed Bayesian calibration against the conventional optimization based approach. In particular, in MC simulation the parameters to be calibrated are used to assess the performance; while in real-world experiment, the measurable canopy cover is adopted to assess the performance.

\subsection{Monte Carlo simulation verification}

Numerical Monte Carlo simulations are firstly conducted to evaluate the parameter estimation performance. Following the exiting literature [17, 36, 37, variance-based Extended Fourier Amplitude Sensitivity Test (EFAST) is adopted to identify the sensitive parameters of AquaCrop model under different stresses. Then a ten-dimensional parameter vector, highly sensitive to CC and biomass, are selected

$$
\theta=[s t i, p s e, w p, c g c, c c x, m a t, e m e, k c b, c d c, p o p]^{T} \text {. }
$$

The parameter definition and prior interval information are shown in Table 3 . The default parameter values in AquaCrop-OS model are set to be truth.

To represent the noisy observation, the groundtruth CC data is added with a Gaussian measurement noise with zero mean and a variance of $0.0005^{2}$. The time period of simulation is consistent with the experiment period, which is from $05 /$ October/2017 to 05/June/2018 and the data acquisition interval is 10 days. The simulation iteration is set as 5000, besides, 100 Monte Carlo experiments with random initial value and random noises is performed to test the robustness of both Bayesian and optimisation based calibration methods. 
Table 3: Sensitive parameters with prior information for Monte Carlo simulation.

\begin{tabular}{ccc}
\hline Parameters & Prior Information & Meaning \\
sti & $(10,20)$ & Minimum growing degree days (degC/day) required for full biomass production \\
$w p$ & $(0.5,1)$ & Upper soil water depletion threshold for water stress effects on canopy senescence \\
$c g c$ & $(30,40)$ & Water productivity normalized for $E T_{0}$ and $C 0_{2}(\mathrm{~g} / \mathrm{m} 2)$ \\
$c c x$ & $(0.005,0.02)$ & Canopy growth coefficient \\
$m a t$ & $(1000,2500)$ & Maximum canopy cover fraction \\
eme & $(60,100)$ & Growing degree days from sowing to maturity \\
$k c b$ & $(0.77,1.43)$ & Crop coefficient when canopy growth is complete but prior to senescence \\
$c d c$ & $(0,0.02)$ & Canopy decline coefficient \\
$p o p$ & $(65000,85000)$ & Number of plants per hectare
\end{tabular}

\subsection{Experimental evaluation}

In addition to MC simulation for parameter estimation, experimental validation is further considered. In this case, the time-series CC values learnt from multi-spectral image are used to estimate the uncertain parameters of AquaCrop-OS model. In order to test the capability of the developed algorithm, the prior information in Table 3 is reduced by increasing the uncertain parameter ranges as shown in Table 4 . The iteration is also increased to 6000 to guarantee the convergence, this is because different from MC simulation fewer number of measurements are available in real-world experiments. The remaining settings of MCMC algorithm are the same as MC simulation.

\begin{tabular}{cc|cc}
\multicolumn{4}{c}{ Table 4: Sensitive parameters with prior information for experimental evaluation. } \\
\hline Parameters & Prior Information & Parameters & Prior information \\
\hline sti & $(3,20)$ & mat & $(1500,3250)$ \\
pse & $(0.35,1.85)$ & eme & $(30,250)$ \\
$w p$ & $(5,40)$ & $k c b$ & $(0.5,2.8)$ \\
$c g c$ & $(0.004,0.02)$ & $c d c$ & $(0,0.06)$ \\
$c c x$ & $(0.82,0.99)$ & pop & $(65000,95000)$ \\
\hline
\end{tabular}

\section{Results}

This section presents the comparative results. For MC simulation, parameter estimation performance is quantified in terms of mean estimation and root mean squared error (RMSE). While in experimental evaluation, RMSE is firstly calculated for CC estimation, and the estimated parameter posterior distributions are also shown. 


\subsection{Results of $M C$ simulation}

Monte Carlo analysis with random initial values and various noises is first performed for both Bayesian and optimization approaches. For each MC simulation of the Bayesian approach, a Markov chain is constructed by using MCMC, based on which the parameter estimation is calculated as the mean of the chain. Then mean parameter estimation of the $100 \mathrm{MC}$ simulations are calculated to asses the algorithm stability. On this basis, the estimation error is defined by the following formula.

$$
E_{o p t}=\frac{\left|p_{o p t}-p_{t}\right|}{p_{t}} * 100 \%, E_{b a y}=\frac{\left|p_{b a y}-p_{t}\right|}{p_{t}} * 100 \%
$$

where $E_{o p t}$ and $E_{b a y}$ denote the parameter estimation errors by optimisation and Bayesian methods, respectively. $p_{o p t}$ and $p_{\text {bay }}$ represent the average calibrated parameters with $p_{t}$ being the ground truth. The parameter estimations and their error percentages are shown in Table 5

Table 5: Mean of the estimated parameters and errors for $100 \mathrm{MC}$ runs against ground truth via various methods

\begin{tabular}{cccc} 
Parameters & Bayesian (error\%) & Optimisation (error\%) & Groundtruth \\
\hline sti & $12.7658(6.38)$ & $14.8735(23.9)$ & 12 \\
$p s e$ & $0.7066(2.41)$ & $0.7172(3.94)$ & 0.69 \\
$w p$ & $34.3915(2.05)$ & $35.6457(5.77)$ & 33.7 \\
$c g c$ & $0.0126(0.82)$ & $0.0125(0.05)$ & 0.0125 \\
$c c x$ & $0.9625(0.26)$ & $0.9539(0.63)$ & 0.96 \\
$m a t$ & $1736(2.14)$ & $1845(8.54)$ & 1700 \\
$e m e$ & $82.0408(2.55)$ & $84.0569(5.07)$ & 80 \\
$k c b$ & $1.0154(3.29)$ & $1.0649(1.42)$ & 1.05 \\
$c d c$ & $0.0102(1.90)$ & $0.0100(0.07)$ & 0.01 \\
pop & $75239(0.3185)$ & $75933(1.240)$ & 7500 \\
\hline
\end{tabular}

It follows from Table 5 that in term of parameter estimation Bayesian approach outperforms optimization approach for all parameters except $c g c, k c b$, and $c d c$. The performance is further quantified by using RMSE for the $100 \mathrm{MC}$ runs, where the results are displayed in Table 6. Similarly, it can be seen that Bayesian approach outperforms optimization approach for all parameter estimation in term of RMSE except the parameter eme. 
Table 6: RMSE of 100 Monte Carlo simulations via different methods

\begin{tabular}{ccc|ccc}
\hline \multirow{2}{*}{ Parameters } & Bayesian & Optimisation & Parameters & Bayesian & Optimisation \\
\hline sti & 0.9814 & 4.0684 & mat & 60.3164 & 411.1170 \\
pse & 0.0309 & 0.1361 & $e m e$ & 67.2370 & 65.1902 \\
$w p$ & 0.9066 & 3.6541 & $k c b$ & 0.0513 & 0.1942 \\
$c g c$ & 0.0001 & 0.0004 & $c d c$ & 0.0002 & 0.0031 \\
$c c x$ & 0.0038 & 0.0317 & $p o p$ & 1745 & 6075 \\
\hline
\end{tabular}

\subsection{Results of experimental validation}

In this section, experimental validation is conducted to further evaluate the performance. In particular, the key state $\mathrm{CC}$ is adopted to validate the calibration accuracy. In order to avoid the problem of overfitting, k-fold cross validation is adopted for the time-series data. The 8 experimental $\mathrm{CC}$ values are divided into $\mathrm{k}=4$ disjoint folds of equal size, where k-1 folds are for training and the remaining 1-fold is for testing [38. Considering the particular characteristics of the calibration problem in this study that observation data of the key stages should be preserved for calibration, the dataset is divided into the particular $\mathrm{k}$ folds as shown in Fig 8 . For example, when $k=1$ is chosen for validation, the remaining ones are then for calibration so that the parameters can be estimated along with the predicted CC values. This process is repeated for all four calibration/validation combinations.

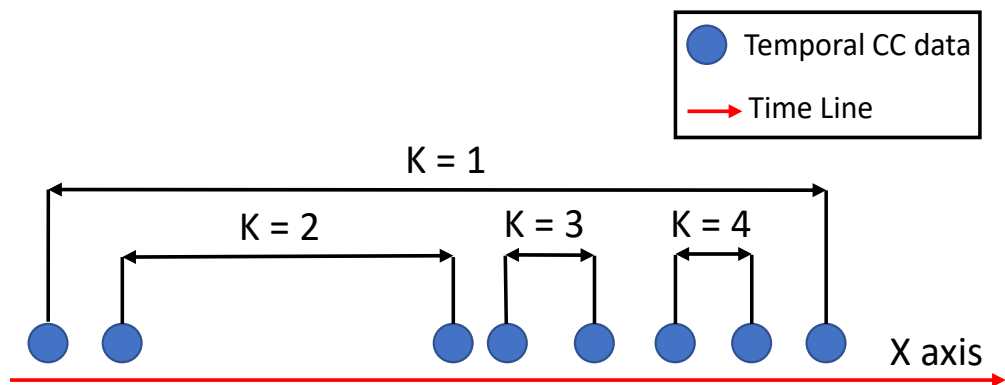

Figure 8: Conceptual explanation of K-fold cross validation datasets.

\subsubsection{Markov Chain and Parameter Estimation}

An example ( $k=2,3,4$ folds for calibration and $k=1$ fold for validation) is illustrated in this part. In Bayesian parameter estimation, the aim is to estimate the posterior probability distribution of parameters given observations rather than a point estimate. By eliminating the burn-time (10\% of the samples) in Markov Chain, it can be seen from Fig 9 that all Markov chains converge to the corresponding equilibrium. Therefore, the posterior probability density distribution of each parameters is reliable.

From the Markov Chain samples, the posterior distribution for each parameter can be represented by a histogram. The normalized probability density of each estimated parameter with original prior information (red line) is displayed 

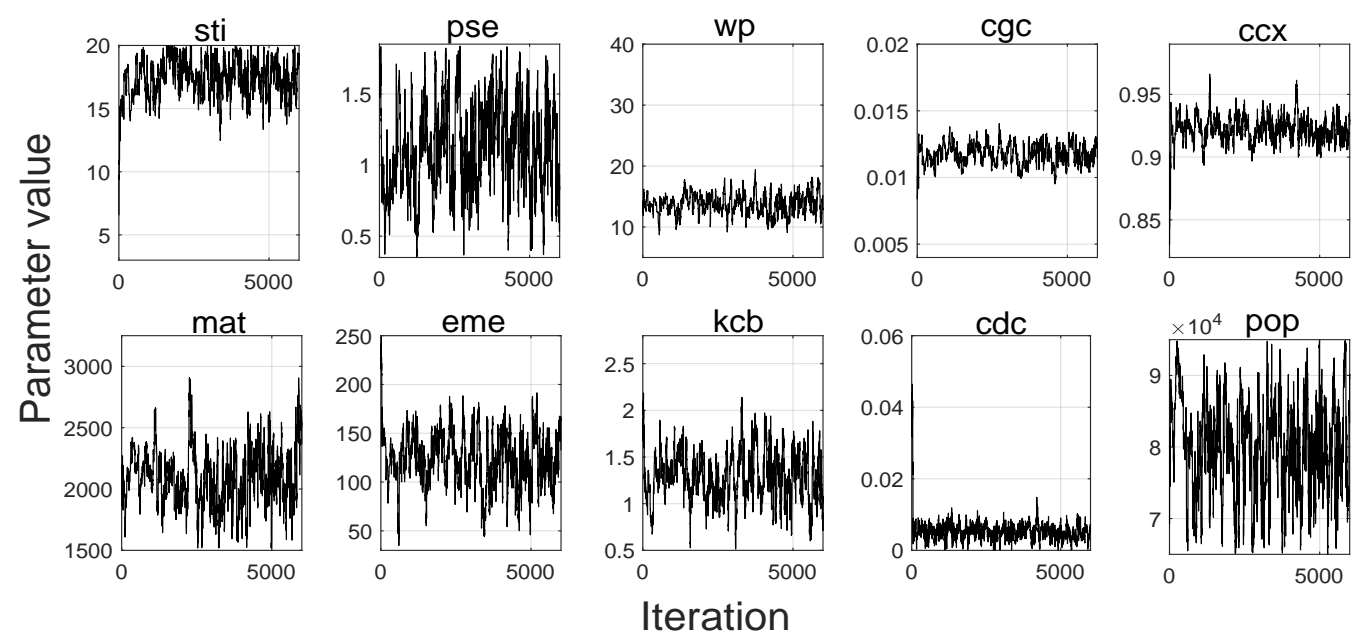

Figure 9: Markov Chain of each parameter using $k=2,3,4$ data for calibration
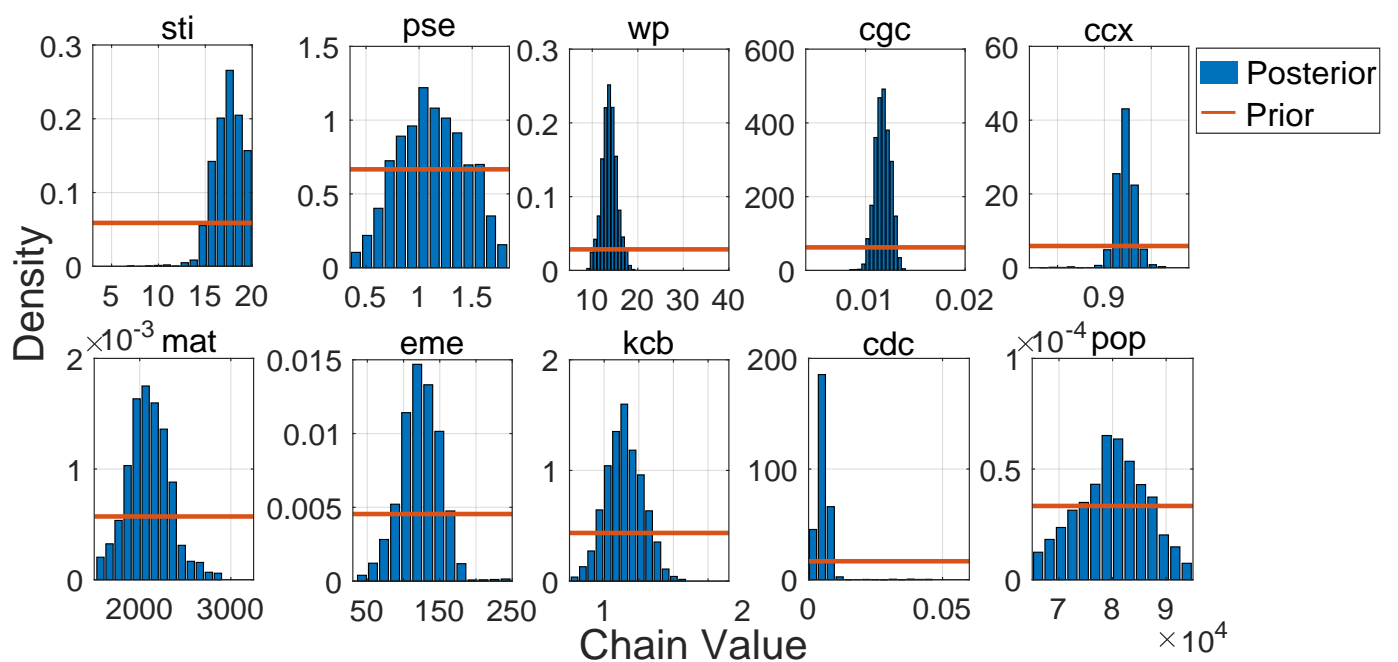

Figure 10: Normalized probability distribution of each parameter using $k=2,3,4$ folds data for calibration.

in Fig 10. It can be seen that the uniform prior distribution has been transformed into posterior distribution by integrating the measurements into the AquaCrop crop model. From the distributions, parameter estimate (e.g. mean, mode) can be derived and more importantly the confidence of parameter estimation can also be quantified. The confidence rule is that the less spread the distribution is, the more reliable the parameter estimation is. However, the optimization based approach can only provide a point estimate without confidence information (see, Fig 11).

It can also be seen from Fig 10 that pse and pop are with a large variance. There exist several possible reasons. First, it may be due to the lack of calibration data in the sensitive growth stages. Secondly, the number of observations may be not enough for the estimate of 10 dimensional parameter vector. The estimated parameters for both Bayesian (e.g. mean value) and optimization (e.g. point estimate) methods are calculated and shown in Table 7 which are used for CC prediction in Section 5.2 .2 . 

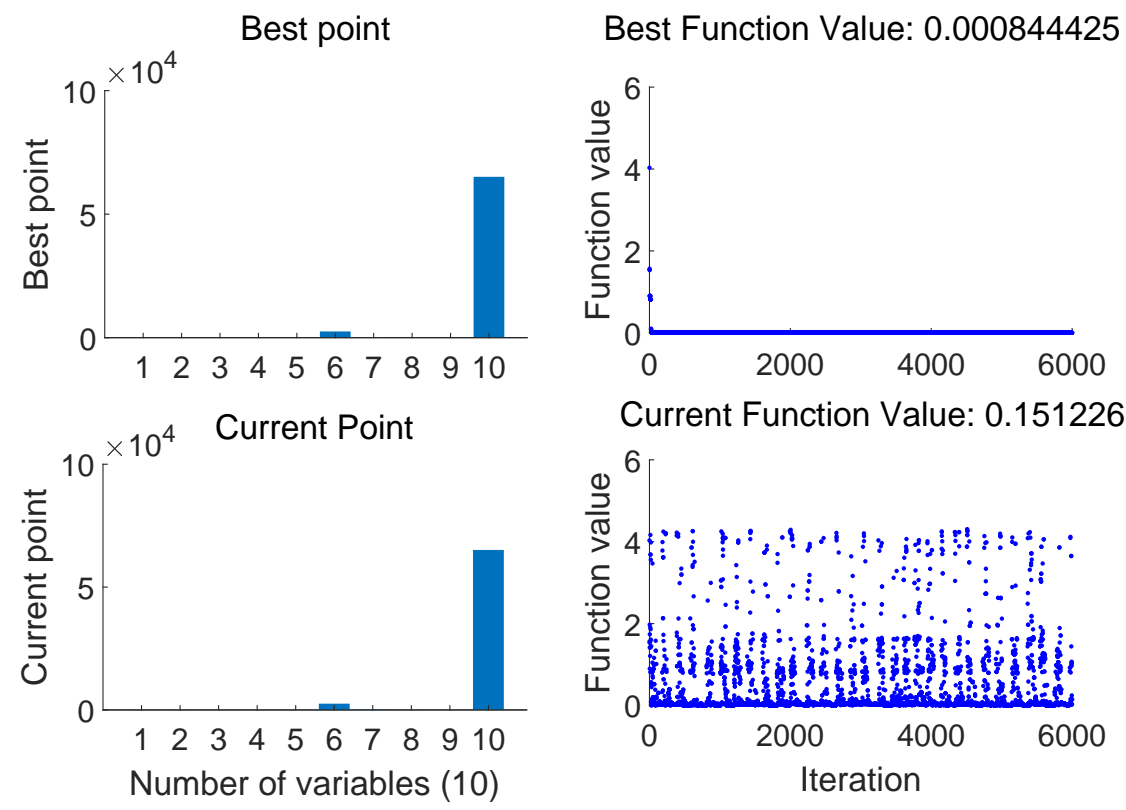

Current Function Value: 0.151226

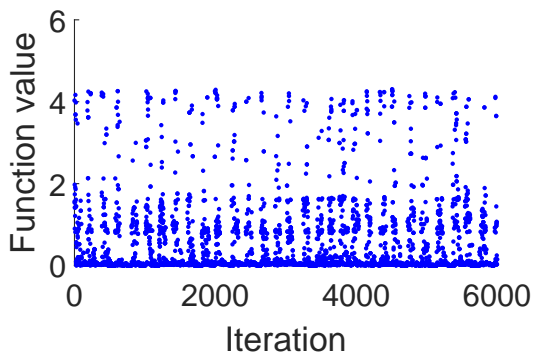

Figure 11: Simulated Annealing based parameter point estimate.

Table 7: Estimated parameters by both Bayesian and optimization methods

\begin{tabular}{ccc|ccc}
\hline Parameters & Bayesian & Optimisation & Parameters & Bayesian & Optimisation \\
\hline$s t i$ & 17.3898 & 19.7103 & mat & 2096 & 2518 \\
$p s e$ & 1.1206 & 0.8055 & eme & 123.3688 & 171.1030 \\
$w p$ & 13.7527 & 11.8404 & $k c b$ & 1.2902 & 2.7902 \\
$c g c$ & 0.0117 & 0.0135 & $c d c$ & 0.0055 & 0.0059 \\
$c c x$ & 0.9221 & 0.9267 & pop & 79900 & 65117 \\
\hline
\end{tabular}

\subsubsection{CC estimation}

The CC estimation over the whole growth season by using both Bayesian and SA optimization approaches is conducted, where the results under different datasets for calibration are displayed in Fig 12. In particular, the coloured lines denote the estimated CC curve for each day.

It can be seen that both approaches can obtain a relatively smooth CC estimate. However, in comparison to SA optimization approach, Bayesian approach obtains a more reliable results when different calibration datasets are adopted. However, when $k=1,2,4$ folds data are chosen for calibration, optimization based approach leads to a poor CC estimate, which substantially deviates from groundtruth data. The main reason is that optimization approach aims at minimizing the error between measurement data and model output data, which will result in poor performance (e.g. local minima due to the complex optimization problem, poor generalization due to the problem of overfitting) when inappropriate observations are chosen. While if no sufficient dataset is available for Bayesian approach, one can easily observe this by inspecting the parameter estimation confidence (e.g. the spread of the parameter distribution). 

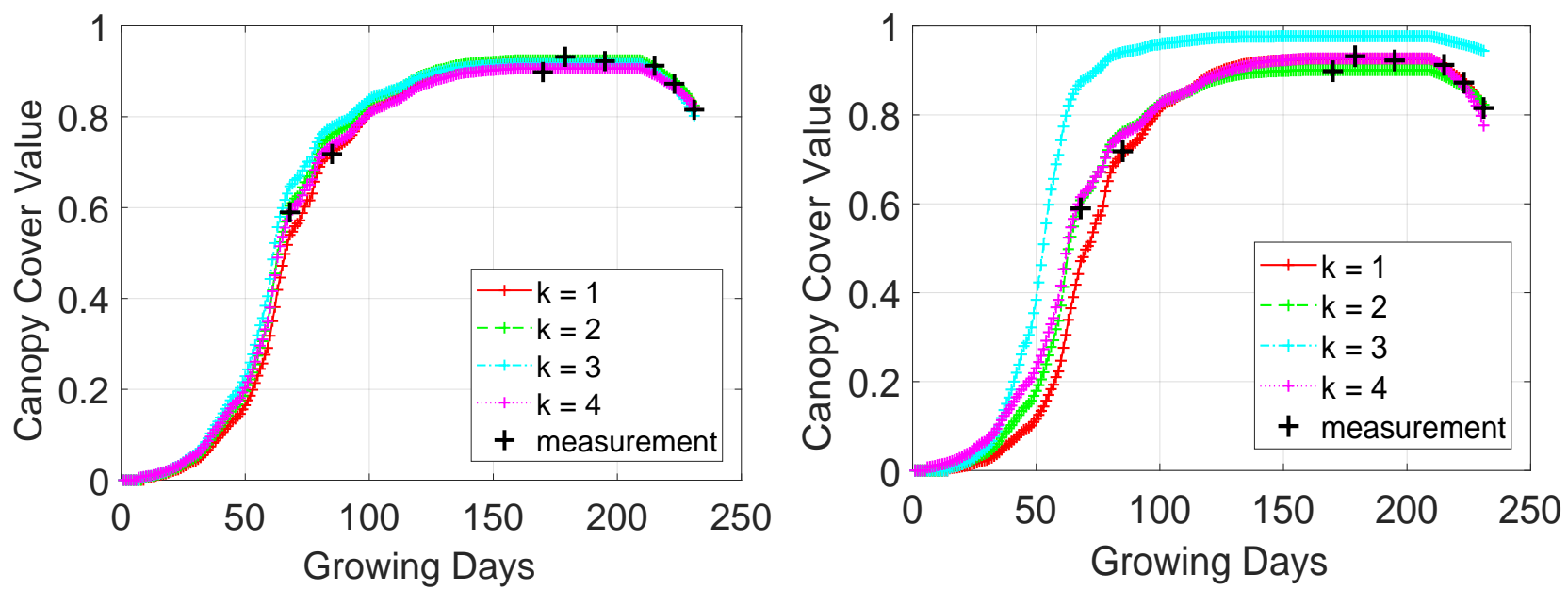

Figure 12: Canopy cover estimation of Bayesian method(left) and optimisation method(right) using different calibration $k$ dataset

Compared to field observations, it can also be seen that Bayesian calibration, building a predictive model by fusing field observations and crop growth model, can also provide CC prediction for days when field observation are unavailable.

\subsubsection{Regression analysis}

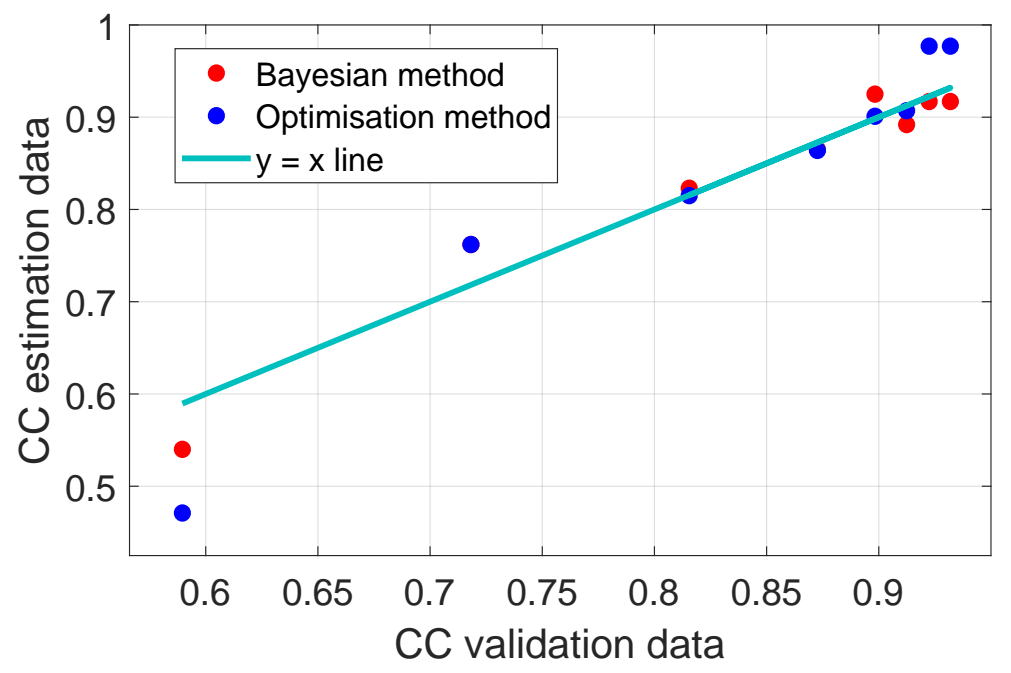

Figure 13: Comparison of estimated CC and validation CC in 2017-2018 year.

The CC estimation against ground truth CC data for different approaches under different datasets for calibration is also displayed in Fig 13 X-axis and y-axis represent the ground truth validation data and the estimated $\mathrm{CC}$ values; the red and blue points represent the estimated CC by using MCMC Bayesian and SA optimization approaches. It can be visually seen that the results of Bayesian approach are closer to $y=x$ line than SA optimization approach.

The RMSE values are also summarised for two approaches in Table 8. It can also be seen that MCMC Bayesian approach results in a smaller RMSE value (in comparison with validation CC) than SA optimization approach. 
Table 8: Summary of optimisation and Bayesian based calibration: regression results

\begin{tabular}{cccc} 
Method & Sensor & Dynamic States & RMSE \\
\hline Bayesian & RedEdge Camera & Canopy Cover & 0.0271 \\
\hline Optimisation & RedEdge Camera & Canopy Cover & 0.0514 \\
\hline
\end{tabular}

\section{Conclusion and future work}

This study introduces a Bayesian framework to assimilate UAV remote sensing images into AquaCrop model so that a more reliable crop model is obtained for crop monitoring. High spatial/spectral multispectral images are first used to calculate the canopy cover by using supervised classification algorithms. Then the remote sensing information is accommodated by Markov Chain Monte Carlo so that the posterior parameter distributions are obtained. Then a systematic validation is conducted, which include Monte Carlo simulations to assess parameter estimation performance and experimental 4-fold cross validation to evaluate canopy cover prediction performance. The Bayesian approach is also compared against the widely used optimization based approach. Comparative results show that both approaches are capable of estimating sensitive parameters and predicting canopy cover with a high accuracy. However, only point estimate is obtained by optimization approach, while Bayesian approach can return parameter posterior distribution reflecting estimation confidence. Bayesian approach also obtains a smaller root mean square error for parameter estimation and canopy cover prediction than optimization based approach. In addition, Bayesian approach is less sensitive to the selection of data points for calibration. Although the results are very promising, there is also room for further improvement, which are summarized as below.

(1) This work is mainly focused on algorithm development and its initial validation by using a small field, algorithm validation by large fields will be more convincing;

(2) More advanced Bayesian inference algorithms can be developed to further improve the performance (e.g. reducing the computation load).

\section{Acknowledgements}

This work was supported by Science and Technology Facilities Council (STFC) under Newton fund with grant number ST/N006852/1. Xi'an Tongfei Aviation Technology Co., Ltd was also acknowledged for their professional support in flying UAV for data collection.

\section{References}

[1] X. Jin, L. Kumar, Z. Li, X. Xu, G. Yang, and J. Wang, "Estimation of winter wheat biomass and yield by combining the aquacrop model and field hyperspectral data," Remote Sensing, vol. 8, no. 12, p. 972, 2016. 
[2] Z. Jiang, Z. Chen, J. Chen, J. Liu, J. Ren, Z. Li, L. Sun, and H. Li, "Application of crop model data assimilation with a particle filter for estimating regional winter wheat yields," IEEE Journal of Selected Topics in Applied Earth Observations and Remote Sensing, vol. 7, no. 11, pp. 4422-4431, 2014.

[3] M. Maki, K. Sekiguchi, K. Homma, Y. Hirooka, and K. Oki, "Estimation of rice yield by simriw-rs, a model that integrates remote sensing data into a crop growth model," Journal of Agricultural Meteorology, vol. 73, no. 1, pp. 2-8, 2017.

[4] X. Jin, L. Kumar, Z. Li, H. Feng, X. Xu, G. Yang, and J. Wang, "A review of data assimilation of remote sensing and crop models," European Journal of Agronomy, vol. 92, pp. 141-152, 2018.

[5] F. Liu, X. Liu, L. Zhao, C. Ding, J. Jiang, and L. Wu, "The dynamic assessment model for monitoring cadmium stress levels in rice based on the assimilation of remote sensing and the wofost model," IEEE Journal of Selected Topics in Applied Earth Observations and Remote Sensing, vol. 8, no. 3, pp. 1330-1338, 2015.

[6] N. Brisson, C. Gary, E. Justes, R. Roche, B. Mary, D. Ripoche, D. Zimmer, J. Sierra, P. Bertuzzi, P. Burger, et al., "An overview of the crop model stics," European Journal of agronomy, vol. 18, no. 3-4, pp. 309-332, 2003.

[7] P. Steduto, T. C. Hsiao, D. Raes, and E. Fereres, "Aquacropthe fao crop model to simulate yield response to water: I. concepts and underlying principles," Agronomy Journal, vol. 101, no. 3, pp. 426-437, 2009.

[8] T. Foster, N. Brozović, A. Butler, C. Neale, D. Raes, P. Steduto, E. Fereres, and T. C. Hsiao, "Aquacrop-os: An open source version of fao's crop water productivity model," Agricultural water management, vol. 181, pp. 18-22, 2017.

[9] T. Zhang, J. Su, C. Liu, and W.-H. Chen, "Bayesian calibration of aquacrop model," in 2018 37th Chinese Control Conference (CCC), pp. 10334-10339, IEEE, 2018.

[10] T.-X. Zhang, J.-Y. Su, C.-J. Liu, and W.-H. Chen, "Potential bands of sentinel-2a satellite for classification problems in precision agriculture," International Journal of Automation and Computing, vol. 16, no. 1, pp. 16-26, 2019.

[11] J. Su, C. Liu, M. Coombes, X. Hu, C. Wang, X. Xu, Q. Li, L. Guo, and W.-H. Chen, "Wheat yellow rust monitoring by learning from multispectral uav aerial imagery," Computers and Electronics in Agriculture, vol. 155, pp. 157-166, 2018.

[12] J. Su, C. Liu, X. Hu, X. Xu, L. Guo, and W.-H. Chen, "Spatio-temporal monitoring of wheat yellow rust using uav multispectral imagery," Computers and Electronics in Agriculture, accepted, 2019.

[13] L. Wallace, A. Lucieer, and C. S. Watson, "Evaluating tree detection and segmentation routines on very high resolution uav lidar data," IEEE Transactions on Geoscience and Remote Sensing, vol. 52, no. 12, pp. 7619-7628, 2014.

[14] L. Shi, S. Hu, and Y. Zha, "Estimation of sugarcane yield by assimilating uav and ground measurements via ensemble kalman filter," in IGARSS 2018-2018 IEEE International Geoscience and Remote Sensing Symposium, pp. 8816-8819, IEEE, 2018.

[15] G. Ma, J. Huang, W. Wu, J. Fan, J. Zou, and S. Wu, "Assimilation of modis-lai into the wofost model for forecasting regional winter wheat yield," Mathematical and Computer Modelling, vol. 58, no. 3-4, pp. 634-643, 2013. 
[16] Y. Huang, Y. Zhu, W. Li, W. Cao, and Y. Tian, "Assimilating remotely sensed information with the wheatgrow model based on the ensemble square root filter for improving regional wheat yield forecasts," Plant Production Science, vol. 16, no. 4, pp. 352-364, 2013.

[17] X. Jin, Z. Li, C. Nie, X. Xu, H. Feng, W. Guo, and J. Wang, "Parameter sensitivity analysis of the aquacrop model based on extended fourier amplitude sensitivity under different agro-meteorological conditions and application," Field Crops Research, vol. 226, pp. 1-15, 2018.

[18] A. C. Fiala, S. L. Garman, and A. N. Gray, "Comparison of five canopy cover estimation techniques in the western oregon cascades," Forest ecology and management, vol. 232, no. 1-3, pp. 188-197, 2006.

[19] E. Hamuda, M. Glavin, and E. Jones, "A survey of image processing techniques for plant extraction and segmentation in the field," Computers and Electronics in Agriculture, vol. 125, pp. 184-199, 2016.

[20] J. Torres-Sánchez, J. M. Peña, A. I. de Castro, and F. López-Granados, "Multi-temporal mapping of the vegetation fraction in early-season wheat fields using images from uav," Computers and Electronics in Agriculture, vol. 103, pp. 104-113, 2014.

[21] J. A. Vrugt, H. V. Gupta, L. A. Bastidas, W. Bouten, and S. Sorooshian, "Effective and efficient algorithm for multiobjective optimization of hydrologic models," Water Resources Research, vol. 39, no. 8, 2003.

[22] J.-H. Jeon, C.-G. Park, and B. A. Engel, "Comparison of performance between genetic algorithm and sce-ua for calibration of scs-cn surface runoff simulation," Water, vol. 6, no. 11, pp. 3433-3456, 2014.

[23] X. Jin, G. Yang, Z. Li, X. Xu, J. Wang, and Y. Lan, "Estimation of water productivity in winter wheat using the aquacrop model with field hyperspectral data," Precision Agriculture, vol. 19, no. 1, pp. 1-17, 2018.

[24] S. Whyte, C. Walsh, and J. Chilcott, "Bayesian calibration of a natural history model with application to a population model for colorectal cancer," Medical decision making, vol. 31, no. 4, pp. 625-641, 2011.

[25] M. Van Oijen, J. Rougier, and R. Smith, "Bayesian calibration of process-based forest models: bridging the gap between models and data," Tree Physiology, vol. 25, no. 7, pp. 915-927, 2005.

[26] J. Su, M. Coombes, C. Liu, Y. Zhu, X. Song, S. Fang, L. Guo, and W.-H. Chen, "Machine learning based crop drought mapping system by uav remote sensing rgb imagery," Unmanned Systems, 2018, DOI: 10.1142/S2301385020500053.

[27] A. Ozturk, A. Unlukara, A. Ipek, and B. Gurbuz, "Effects of salt stress and water deficit on plant growth and essential oil content of lemon balm (melissa officinalis 1.)," Pak. J. Bot, vol. 36, no. 4, pp. 787-792, 2004.

[28] J. Su, D. Yi, C. Liu, L. Guo, and W.-H. Chen, "Dimension reduction aided hyperspectral image classification with a small-sized training dataset: experimental comparisons," Sensors, vol. 17, no. 12, p. 2726, 2017.

[29] C. Thornton, F. Hutter, H. H. Hoos, and K. Leyton-Brown, "Auto-weka: Combined selection and hyperparameter optimization of classification algorithms," in Proceedings of the 19th ACM SIGKDD international conference on Knowledge discovery and data mining, pp. 847-855, ACM, 2013. 
[30] P. Silvestro, S. Pignatti, S. Pascucci, H. Yang, Z. Li, G. Yang, W. Huang, and R. Casa, "Estimating wheat yield in china at the field and district scale from the assimilation of satellite data into the aquacrop and simple algorithm for yield (safy) models," Remote Sensing, vol. 9, no. 5, p. 509, 2017.

[31] A. Kanso, M.-C. Gromaire, E. Gaume, B. Tassin, and G. Chebbo, "Bayesian approach for the calibration of models: application to an urban stormwater pollution model," Water Science and Technology, vol. 47, no. 4, pp. 77-84, 2003.

[32] M. Van Oijen, C. Reyer, F. Bohn, D. Cameron, G. Deckmyn, M. Flechsig, S. Härkönen, F. Hartig, A. Huth, A. Kiviste, et al., "Bayesian calibration, comparison and averaging of six forest models, using data from scots pine stands across europe," Forest Ecology and Management, vol. 289, pp. 255-268, 2013.

[33] M. T. Wentworth, R. C. Smith, and B. Williams, "Bayesian model calibration and uncertainty quantification for an hiv model using adaptive metropolis algorithms," Inverse Problems in Science and Engineering, vol. 26, no. 2, pp. 233-256, 2018.

[34] H. Haario, M. Laine, A. Mira, and E. Saksman, "Dram: efficient adaptive mcmc," Statistics and computing, vol. 16, no. 4, pp. 339-354, 2006.

[35] L. M. Păun, M. U. Qureshi, M. Colebank, N. A. Hill, M. S. Olufsen, M. A. Haider, and D. Husmeier, "Mcmc methods for inference in a mathematical model of pulmonary circulation," Statistica Neerlandica, vol. 72, no. 3, pp. 306-338, 2018.

[36] E. Vanuytrecht, D. Raes, and P. Willems, "Global sensitivity analysis of yield output from the water productivity model," Environmental Modelling \&S Software, vol. 51, pp. 323-332, 2014.

[37] H.-m. XING, X.-g. XU, Z.-h. LI, Y.-j. CHEN, H.-k. FENG, G.-j. YANG, and Z.-x. CHEN, "Global sensitivity analysis of the aquacrop model for winter wheat under different water treatments based on the extended fourier amplitude sensitivity test," Journal of Integrative Agriculture, vol. 16, no. 11, pp. 2444-2458, 2017.

[38] T.-T. Wong, "Performance evaluation of classification algorithms by k-fold and leave-one-out cross validation," Pattern Recognition, vol. 48, no. 9, pp. 2839-2846, 2015. 